\title{
Supporting Information for Layered Niobium Oxide Hydrate Anode with Excellent Performance for Lithium-Ion Batteries
}

Fenghao Liang, ${ }^{\dagger}$ Daoning Wu,${ }^{\dagger}$ Lei Jiang,,${ }^{\dagger}$ Zhe Zhang, ${ }^{\dagger}$ Wei Zhang, ${ }^{*},{ }^{\dagger}$ Yichuan Rui,,${ }^{*} \dagger$ Bohejin Tang*,† and Fengjiao Liu*,†

${ }^{\dagger}$ College of Chemistry and Chemical Engineering, Shanghai University of Engineering Science, Shanghai 201620, P.R. China

*Department of Materials Engineering, KU Leuven, Leuven, 3001, Belgium.

${ }^{*}$ Corresponding Author:

wei.zhang@kuleuven.be (W. Zhang), ryc713@126.com (Y. Rui), tangbohejin@sues.edu.cn (B. Tang), fjliu@sues.edu.cn 
Table S1. Comparison of the capacity of present work based on niobium oxide with this work.

\begin{tabular}{|c|c|c|c|c|c|}
\hline Samples & $\begin{array}{l}\text { Current density } \\
\qquad\left(\mathrm{mA} \mathrm{g}^{-1}\right)\end{array}$ & Cycle number & $\begin{array}{l}\text { Capacity } \\
\left(\mathrm{mAh} \mathrm{g}^{-1}\right)\end{array}$ & $\begin{array}{l}\text { Loading amount } \\
\qquad(\mathrm{mg})\end{array}$ & Ref. \\
\hline $\begin{array}{c}\mathrm{T}-\mathrm{Nb}_{2} \mathrm{O}_{5} \\
\text { nanostructures }\end{array}$ & 200 & 100 & 178.2 & & 1 \\
\hline $\begin{array}{c}\mathrm{T}-\mathrm{Nb}_{2} \mathrm{O}_{5} / \text { coal-based } \\
\text { graphite }\end{array}$ & 200 & 200 & 216 & & 2 \\
\hline $\mathrm{Ag}-\mathrm{N}-\mathrm{Nb}_{2} \mathrm{O}_{5}$ & 500 & 500 & 260 & & 3 \\
\hline $\begin{array}{l}\text { tetragonal- } \mathrm{Nb}_{2} \mathrm{O}_{5} \\
\text { microspheres }\end{array}$ & 200 & 1000 & 139 & & 4 \\
\hline $\begin{array}{c}\mathrm{Nb}_{2} \mathrm{O}_{5} / \text { carboxyl } \\
\text { nanotube }\end{array}$ & 100 & 100 & 382 & & 5 \\
\hline $\begin{array}{c}\mathrm{Nb}_{2} \mathrm{O}_{5} \text {-carbon } \\
\text { core-shell } \\
\text { nanocomposite }\end{array}$ & 200 & 300 & 130 & & 6 \\
\hline $\mathrm{Nb}_{2} \mathrm{O}_{5} @ \mathrm{rGO}$ & 1000 & 1000 & 270 & & 7 \\
\hline $\mathrm{Nb}_{2} \mathrm{O}_{5} / \mathrm{rGO}$ & 200 & 100 & 332 & & 8 \\
\hline $\begin{array}{c}\mathrm{Nb}_{2} \mathrm{O}_{5} @ \text { carbon } \\
\text { hollow } \\
\text { microspheres }\end{array}$ & 40 & 100 & 350.15 & & 9 \\
\hline $\mathrm{NbO}_{2} /$ carbon & 200 & 500 & 225 & & 10 \\
\hline $\begin{array}{c}\mathrm{NbO} / \mathrm{Cu} \\
\text { nanocrystalline }\end{array}$ & 100 & 100 & 580 & & 11 \\
\hline nano- $\mathrm{Nb}_{2} \mathrm{O}_{5}$ & 500 & 800 & 143 & & 12 \\
\hline $\begin{array}{c}\text { carbon } / \mathrm{Nb}_{2} \mathrm{O}_{5} \\
\text { microsphere }\end{array}$ & 1000 & 200 & 100 & & 13 \\
\hline $\begin{array}{l}\mathrm{T}-\mathrm{Nb}_{2} \mathrm{O}_{5} / \mathrm{NC} \\
\text { nanosheets }\end{array}$ & 1000 & 1000 & 147.6 & & 14 \\
\hline NOH 50 & 100 & 200 & 1181.66 & 1.19 & \\
\hline NOH 100 & 100 & 200 & 346.19 & 1.19 & This \\
\hline NOH 150 & 100 & 200 & 246.26 & 1.19 & work \\
\hline
\end{tabular}


Table S2. Theoretical calculation of simulated adsorption energy of hydronium ion.

\begin{tabular}{ccccc}
\hline $\begin{array}{c}\text { Energy/ } \\
\text { (a.u.) }\end{array}$ & $\mathrm{E}_{(\mathrm{Li})}$ & $\mathrm{E}_{(\mathrm{H} 3 \mathrm{O}+)}$ & $\mathrm{E}_{(\mathrm{H} 3 \mathrm{O}+@ \mathrm{Li})}$ & $\mathrm{E}_{(\mathrm{ads})}$ \\
\hline H site & -29.72702848 & -76.28060121 & -83.27284654 & 0.24137464 \\
O site & -29.72702848 & -76.28060121 & -83.17270985 & 0.34151133 \\
\hline
\end{tabular}
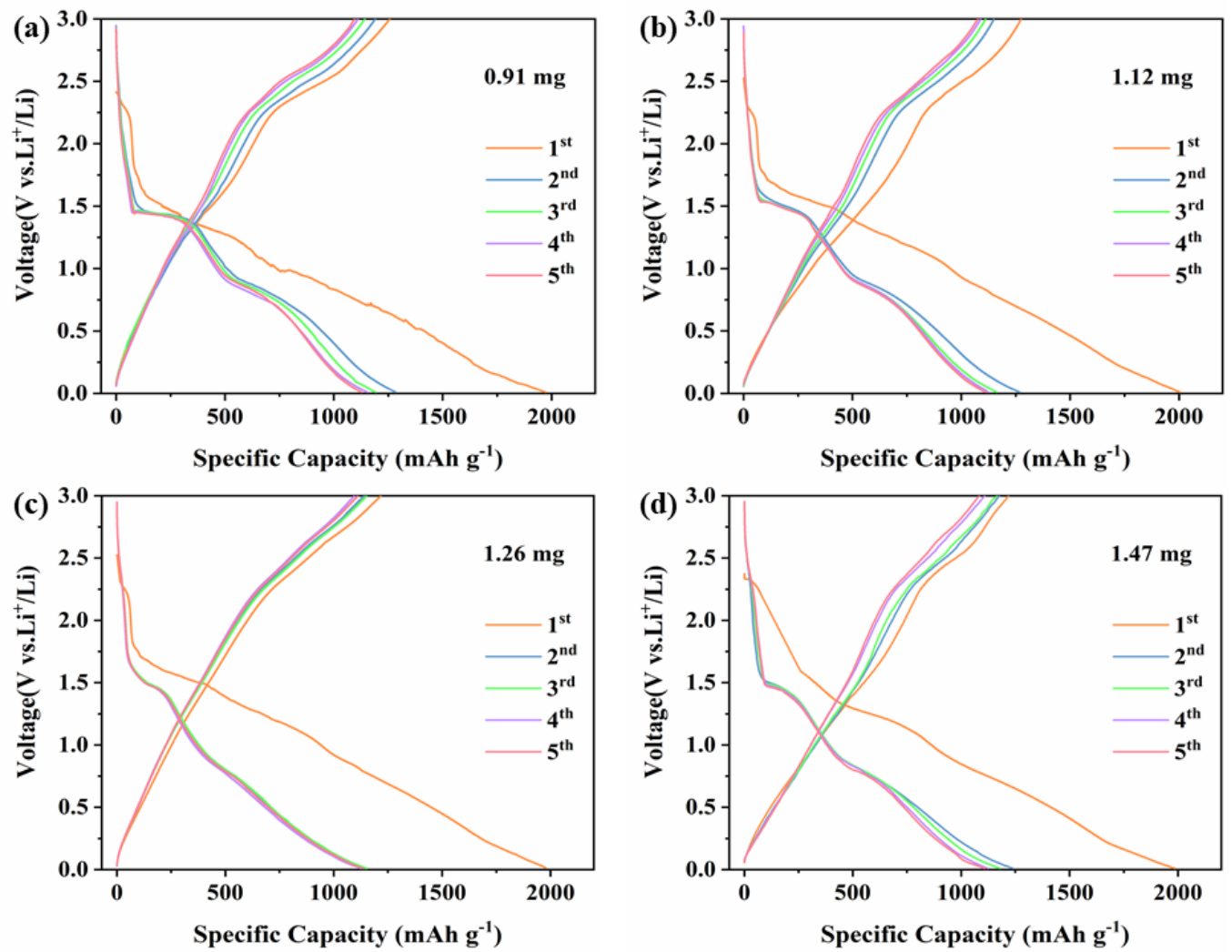

Figure S1. Discharge/charge curves of NOH50 with (a) $0.91 \mathrm{mg}$, (b) $1.12 \mathrm{mg}$, (c) $1.26 \mathrm{mg}$ and (d) $1.47 \mathrm{mg}$ loading amount. 


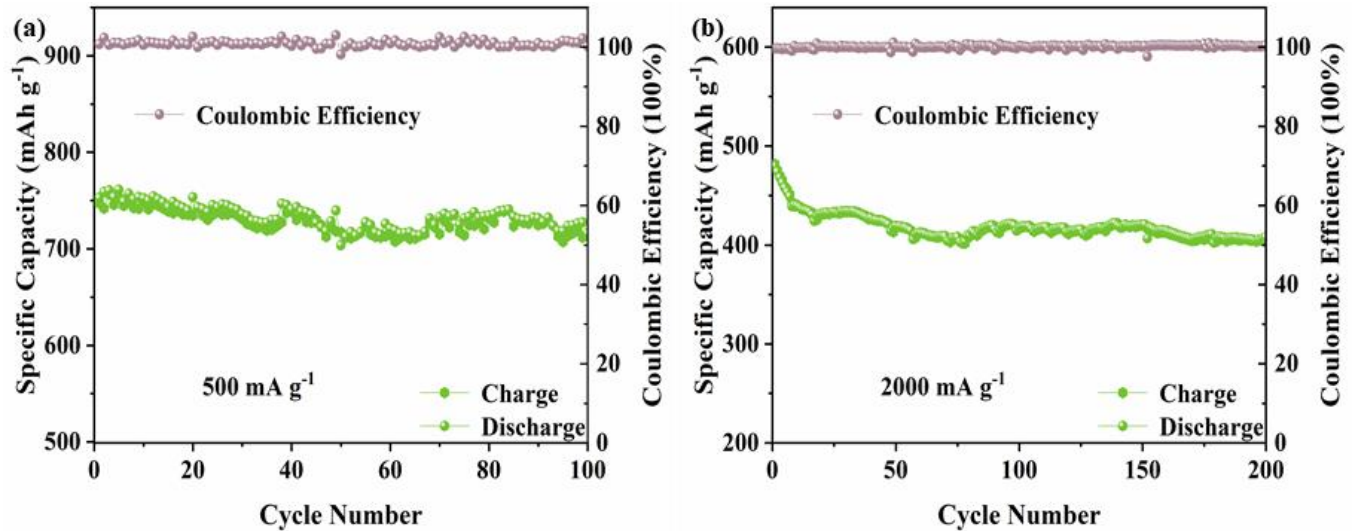

Figure S2. Cycling performance at (a) $500 \mathrm{~mA} \mathrm{~g}^{-1}$ and (b) $2000 \mathrm{~mA} \mathrm{~g}^{-1}$ of $\mathrm{NOH} 50$ anode. 

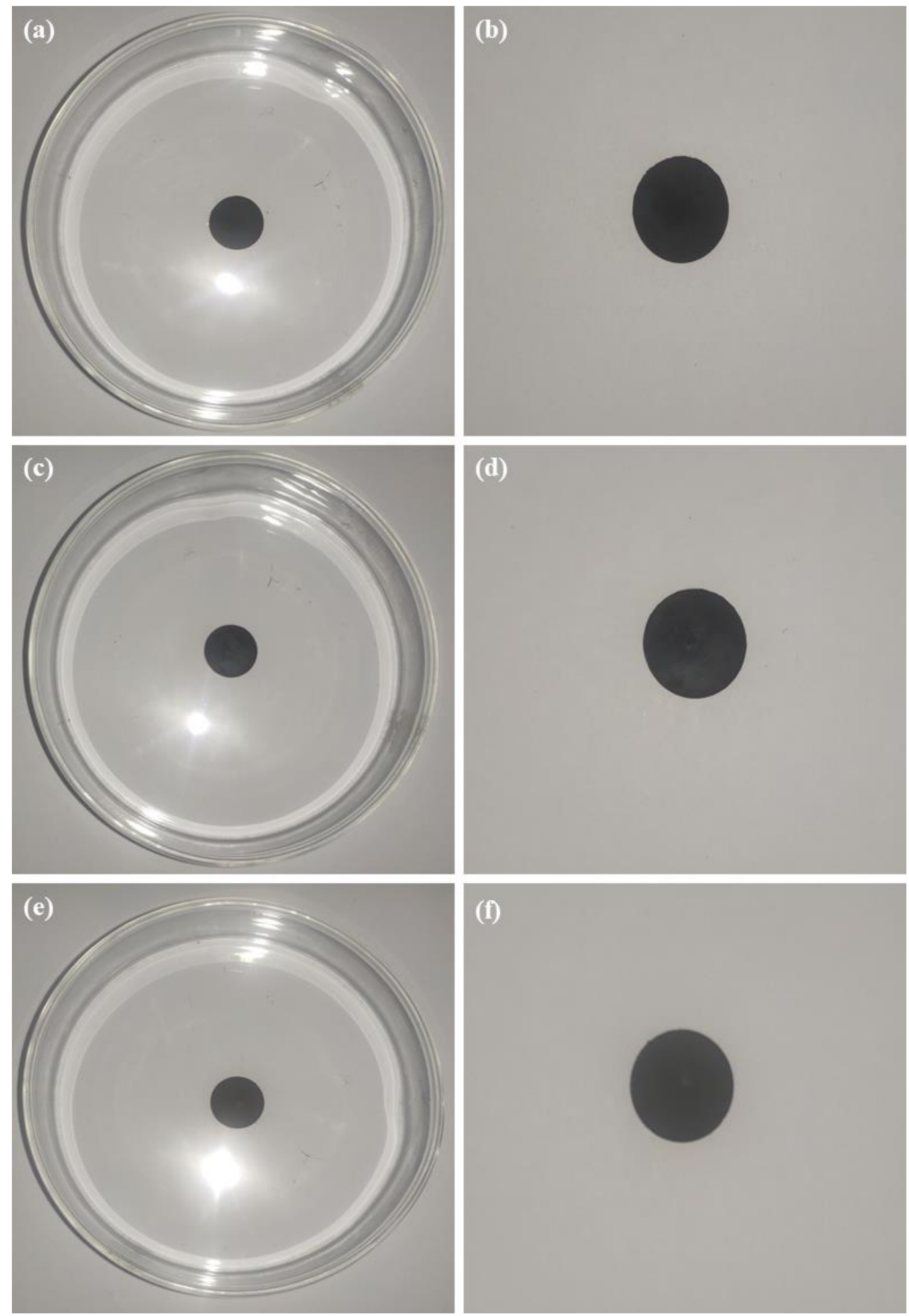

Figure S3. (a, b) NOH 50, (c, d) NOH 100 and (e, f) NOH 150 anode sheet of with copper foil as current collector. 

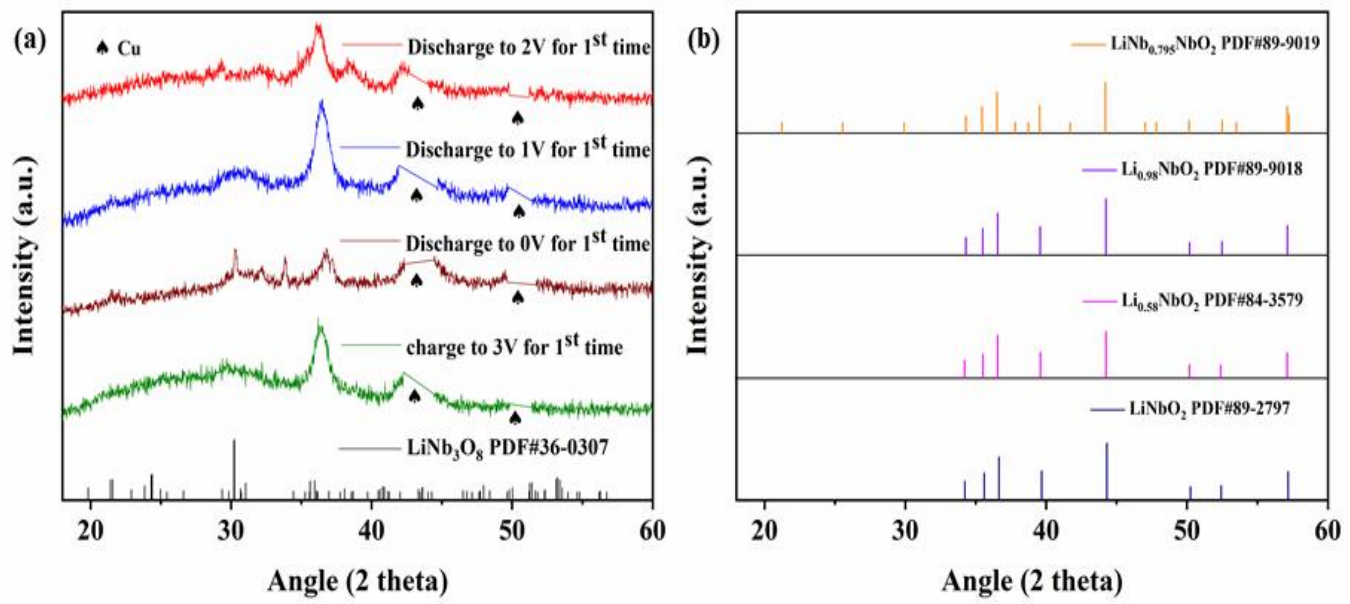

Figure S4. (a) XRD patterns of NOH50 after 1 cycle. (b) PDF standard card of $\mathrm{LiNbO}_{2}, \mathrm{Li}_{0.58} \mathrm{NbO}_{2}, \mathrm{Li}_{0.98} \mathrm{NbO}_{2}$ and $\mathrm{Li}_{0.795} \mathrm{NbO}_{2}$. 


\section{Reference}

(1) Qu, X.; Xing, B.; Huang, G.; Zhao, H.; Jiang, Z.; Zhang, C.; Hong, S. W.; Cao, Y. Facile Synthesis of Flower-Like $\mathrm{T}-\mathrm{Nb}_{2} \mathrm{O}_{5}$ Nanostructures as Anode Materials for Lithium-Ion Battery. J. Mater. Sci.: Mater. Electron. 2020, 32, 875-885.

(2) Qu, X.; Liu, Y.; Li, B.; Xing, B.; Huang, G.; Zhang, C.; Hong, S. W.; Yu, J.; Cao, Y. Synthesis of High Reversibility Anode Composite Materials Using $\mathrm{T}_{-} \mathrm{Nb}_{2} \mathrm{O}_{5}$ and Coal-Based Graphite for Lithium-Ion Battery Applications. Energy Fuels 2020, 34, 3887-3894.

(3) Cheong, J. Y.; Kim, C.; Jung, J.-W.; Yoon, K. R.; Cho, S.-H.; Youn, D.-Y.; Jang, H.-Y.; Kim, I.-D. Formation of a Surficial Bifunctional Nanolayer on $\mathrm{Nb}_{2} \mathrm{O}_{5}$ for Ultrastable Electrodes for Lithium-Ion Battery. Small 2017, 13, No. 1603610.

(4) Hu, Z.; He, Q.; Liu, Z.; Liu, X.; Qin, M.; Wen, B.; Shi, W.; Zhao, Y.; Li, Q.; Mai, L. Facile Formation of Tetragonal- $\mathrm{Nb}_{2} \mathrm{O}_{5}$ Microspheres for High-Rate and Stable Lithium Storage with High Areal Capacity. Sci. Bull. 2020, 65, 1154-1162.

(5) Kang, J.; Zhang, H.; Zhan, Z.; Li, Y.; Ling, M.; Gao, X. Construction of a Flexible $\mathrm{Nb}_{2} \mathrm{O}_{5} /$ Carboxyl Multiwalled Carbon Nanotube Film as Anode for Lithium and Sodium Storages. ACS Appl. Energy Mater. 2020, 3, 11841-11847.

(6) Li, G.; Wang, X.; Ma, X. $\mathrm{Nb}_{2} \mathrm{O}_{5}$-Carbon Core-Shell Nanocomposite as Anode Material for Lithium Ion Battery. J. Energy Chem. 2013, 22, 357-362.

(7) Kang, R.; Li, S.; Zou, B.; Liu, X.; Zhao, Y.; Qiu, J.; Li, G.; Qiao, F.; Lian, J. Design of $\mathrm{Nb}_{2} \mathrm{O}_{5} @ \mathrm{rGO}$ Composites to Optimize the Lithium-Ion Storage Performance. J. Alloys Compd. 2021, 865, No. 158824.

(8) Qu, X.; Liu, Y.; Li, B.; Xing, B.; Huang, G.; Zhao, H.; Jiang, Z.; Zhang, C.; Hong, S. W.; Cao, Y. Nanostructured $\mathrm{T}-\mathrm{Nb}_{2} \mathrm{O}_{5}$-Based Composite with Reduced Graphene Oxide for Improved Performance Lithium-Ion Battery Anode. J. Mater. Sci. 2020, 55, 13062-13074.

(9) Zhou, W.; Fang, L.; Long, L.; Wang, L.; Chen, H.; Li, Y.; Jia, C. Facile Preparation of $\mathrm{Nb}_{2} \mathrm{O}_{5} @$ Carbon Hollow Microspheres as High-Performance Anode Materials for Lithium-Ion Batteries. J. Nanosci. Nanotechnol. 2019, 19, 268-271.

(10) Ji, Q.; Gao, X.; Zhang, Q.; Jin, L.; Wang, D.; Xia, Y.; Yin, S.; Xia, S.; Hohn, N.; Zuo, X.; Wang, X.; Xie, S.; Xu, Z.; Ma, L.; Chen, L.; Chen, G. Z.; Zhu, J.; Hu, B.; Müller $\square$ Buschbaum, P.; Bruce, P. G.; Cheng, Y. J. Dental Resin Monomer Enables Unique $\mathrm{NbO}_{2} /$ Carbon Lithium $\square$ Ion Battery Negative Electrode with Exceptional Performance. Adv. Funct. Mater. 2019, 29, No. 1904961.

(11) Huang, P.; Huang, M.; Hu, H.; Zhong, Y.; Lai, C.; Chou, S. Single Crystal Polyoxoniobate Derived NbO/Cu Nanocrystalline@N-Doped Carbon Loaded onto Reduced Graphene Oxide Enabling High Rate and High Capacity Li/Na Storage. $J$. Mater. Chem. A 2019, 7, 26513-26523.

(12) Lübke, M.; Sumboja, A.; Johnson, I. D.; Brett, D. J. L.; Shearing, P. R.; Liu, Z.; Darr, J. A. High Power Nano- $\mathrm{Nb}_{2} \mathrm{O}_{5}$ Negative Electrodes for Lithium-Ion Batteries. Electrochim. Acta 2016, 192, 363-369.

(13) $\mathrm{Hu}$, J.; Li, J.; Wang, K.; Xia, H. Self-Assembly $\mathrm{Nb}_{2} \mathrm{O}_{5}$ Microsphere with Hollow and Carbon Coated Structure as High Rate Capability Lithium-Ion Electrode 
Materials. Electrochim. Acta 2020, 331, No. 135364.

(14) Jiang, S.; Dong, S.; Wu, L.; Chen, Z.; Shen, L.; Zhang, X. Pseudocapacitive $\mathrm{T}-\mathrm{Nb}_{2} \mathrm{O}_{5} / \mathrm{N}$-Doped Carbon Nanosheets Anode Enable High Performance Lithium-Ion Capacitors. J. Electroanal. Chem. 2019, 842, 82-88. 